\title{
Comparison of European and Asian Morphology
}

\author{
James WEBSTER*, Jeremy CORNOLO \\ Oxylane Research, Villeneuve-d'Ascq, Lille, France
}

\begin{abstract}
http://dx.doi.org/10.15221/13.238

Due to the increase in global brands the effect of geographic origin and racial background on morphology has become increasingly important when developing sizing systems. Previous research has identified that there are significant morphological differences between countries (Lin et al. 2004). It has been suggested that the variations of body dimensions of different groups can be observed in terms of overall body size and bodily proportions (Pheasant, 1996). Therefore, it is crucial for global brands to understand the differences in morphology across the different regions they trade in, and to determine if one sizing system is capable of providing good fit across all regions. This paper aims to determine the key differences between Asian and European populations with regards to key anthropometric measurements.
\end{abstract}

To determine the difference between the two populations, 6000 adult 3D scans were analysed (2200 Chinese, 3800 European (French and Spanish), with 32 body measurements extracted. The data was categorised into 4 groups: Chinese male, Chinese female, EU male and EU female. Initially, the two populations were analysed with regards to the mean, 5th and 95th percentiles, for each of the 32 measures, enabling both the average population and range to be analysed. Secondly, statistical analysis was performed to determine where the key differences between the populations was and if these differences were significant.

The data analysis identified key differences between the morphology of the two populations with regards to both the mean and variation within the population for men and women. Significant differences were found for 14 and 16 of the 32 measures for men and women respectively. Further analysis identified that as well as differences in size, demonstrated by the analysis of individual measures, there were also significant differences in shape. Shape differences were highlighted through the comparison of the ratio of different measures. One example of this was leg length, where the Chinese population were found to have significantly shorter legs, when their overall height was equal to that of their European counterpart.

The results from this study identified that there are several differences between the European and Chinese populations with regards to both shape and size. It was found that the difference between the female populations was larger than that for the men, with a significant difference being found for a greater number of measures. These findings suggest that it is of interest to develop individual sizing systems for each region (Europe and Asia) to accommodate these differences and to maximise user satisfaction.

\section{Introduction}

A representative garment sizing system is essential for effective clothing design and production. The objective of garment sizing is to divide standardized dimensions for the body and garments into categories, with the aim to fit the maximum number of people with the minimum number of sizes. An effective sizing system can increase the coverage of the target population, as well as the resulting user satisfaction with regards to fit. One way to increase the coverage rate is to structure the sizing system based on detailed anthropometric data (Zakaria et al, 2008).

The role of a sizing system within the textile industry has been subject to world wide study since 1921, however, there is still no consensus on how to maximise coverage of a population. This is partly due to the technological advancements within scanning technologies, computer processing and materials, as well as the constant evolution of populations. Originally sizes were formulated based on stature and weight alone (Emanuel et al. 1959), and although this was a significant step towards increasing the fit of mass produced/ ready to wear clothing, it was a long way from ideal, as it did not consider form, body composition or individual body parts. 
This initial advancement led to the development of several methodologies, integrating more measurements, these included KS K 0050 and JIS L 4002, which utilise linear grading between sizes. Although the use of linear grading makes size chart development relatively easy and quick, it generally results in limited coverage of the target population (Chung et al. 2007). As a result several optimization methods have been proposed to generate more representative sizing systems, including nonlinear and integer programming approaches (Tryfos, 1986 and McCulloch et al. 1998), cluster analysis, neural networks, data mining and the decision tree approach (Moon and Nam 2003, She et al. 2002 and Hsu and Wang, 2005). The incorporation of these optimization techniques has been found to increase the fit of a garment by minimizing the distance between the sizing system and the size of an individual. However, current sizing systems still do not accommodate a large percentage of people, resulting in their dissatisfaction with products. Also, the shape of human figure types continues to change mainly due to sedentary lifestyles, dietary habits, immigration patterns and the impact of rising trends that affect body shape ideals. Generally, the most notable differences in body size and shape relate to ethnic diversity, age and gender. As a result of the constant evolution of the human form it is imperative that more detailed and representative sizing systems are developed.

With regards to the effect of geographic origin and racial background. Previous research has identified that there are significant morphological differences between countries (Lin et al. 2004). It has been suggested that the variations of body dimensions of different groups can be observed in terms of overall body size and bodily proportions (Pheasant, 1996).

The understanding of differences in morphology between different populations has become increasingly important as a result of internationalization and globalization of the textile market, with potential new markets expanding over Eastern-European and Asian countries. Due to this globalization of brands it is seminal to understand specific differences in religions, cultures, mentalities and body sizes of the prospective buyers. Alongside this, it is also crucial to regularly update sizing databases to enable a broader understanding of population change with regards to human morphology. To fully explore the variations between populations the integration of $3 \mathrm{D}$ analysis of body form / size must be developed to help determine if subtle but important differences exist outside the standard 32 body measurements. Therefore, this paper aims to explore the differences in body size and shape between Europe and China, determining if it is feasible to utilise a single sizing chart whilst ensuring a good coverage of both populations.

\section{Method}

For this study Chinese and European databases collected between 2005 and 2011 were utilised. Data was collected through a combination of manual measurements and 3D scan data, collected through the use of a TC2 NX16 white light scanner. Initially 32 full body measurements were extracted including both length and circumferential measures. In total 2200 Chinese and 3800 EU adults were included within the study. The data was categorised into 4 distinct groups, based on nationality and sex (Chinese male, Chinese female, EU male and EU female).

The initial analysis focused on determining if individual measures were significantly different for the two populations. The data was analysed with regards to the mean, 5th and 95th percentiles, enabling both the average population and range to be analysed. An additional analysis was developed and incorporated to increase the certainty within the results, this involved running a bootstrap resampling method, producing 10,000 unique samples, for each group. Each sample was analysed in terms of mean and standard deviation and compared with each of the other resamples from the other population e.g. EU male resample 1 was compared to China resample 1:10,000. Finally, $t$ tests were used to identify if the differences in populations were significant.

The second stage of analysis focused on determining if a significant difference in ratio between body parts could be found. For this the ratio of height, arm, leg and trunk length were analysed as well as between circumference measures at the calf, knee, thigh, chest, hip and waist. For this analysis each of the length measures were compared to provide a ratio e.g. between leg length and height to determine if there was a significant difference in these ratios between the 2 populations. The same procedure was followed for the circumferential measures. 


\section{Results}

The initial data analysis identified key morphological differences between the two populations with regards to both the mean and variation within the population, as shown in the example depicted in figure 1 for women, with the male data exhibiting similar trends. T tests used to compare each population for all 32 measures identified that the null hypothesis of there being no significant difference between the two populations was rejected for 14 and 16 of the measures for men and women respectively $(p<0.05)$. Within these differences it was apparent that for both men and women the greatest difference occurred in length measures, in particular all measures related to body segment length, for example, arm length. The greatest differences was in leg and trunk length $(<0.05)$ despite the difference in average height being much smaller than expected.

The comparison of the 2 populations also illustrated that the variation in the populations was significantly different, with the Chinese populations for both men and women exhibiting much less variation for 21 and 23 of the body measures respectively, in particular for chest circumference, where the $90 \%$ confidence interval had a range of $39,5 \mathrm{~cm}$ for China and $48,9 \mathrm{~cm}$ for Europe for the for the female population, with a $8.5 \mathrm{~cm}$ difference in range also being found for men.

As well as differences in individual body measures, the results also identified significant differences in body segment ratios, for example torso length to height. The absolute differences in 8 of the female body ratios are shown in figure 2 . Significant differences were found for 5 of the 8 ratios for men and 6 for women, with the only difference occurring in waist to hip ratio, where it was not found to be significant for the male population $(p<0,05)$. The results indicated that arm length was generally shorter for the Chinese population with regards to torso length, as was leg length when compared to height. With the European population being found to have a shorter torso than their Chinese counterpart of the same height. With regards to leg proportions, the results indicate that the Chinese population has a smaller ratio between thigh and calf circumference, suggesting that the Chinese population have larger calves than Europeans who are of the same thigh circumference. Finally, a significant difference was also found in the waist to hip circumference ratio $(p<0.05)$ for women, with a larger difference between the two measures being found within the European population, indicating a more defined waist.
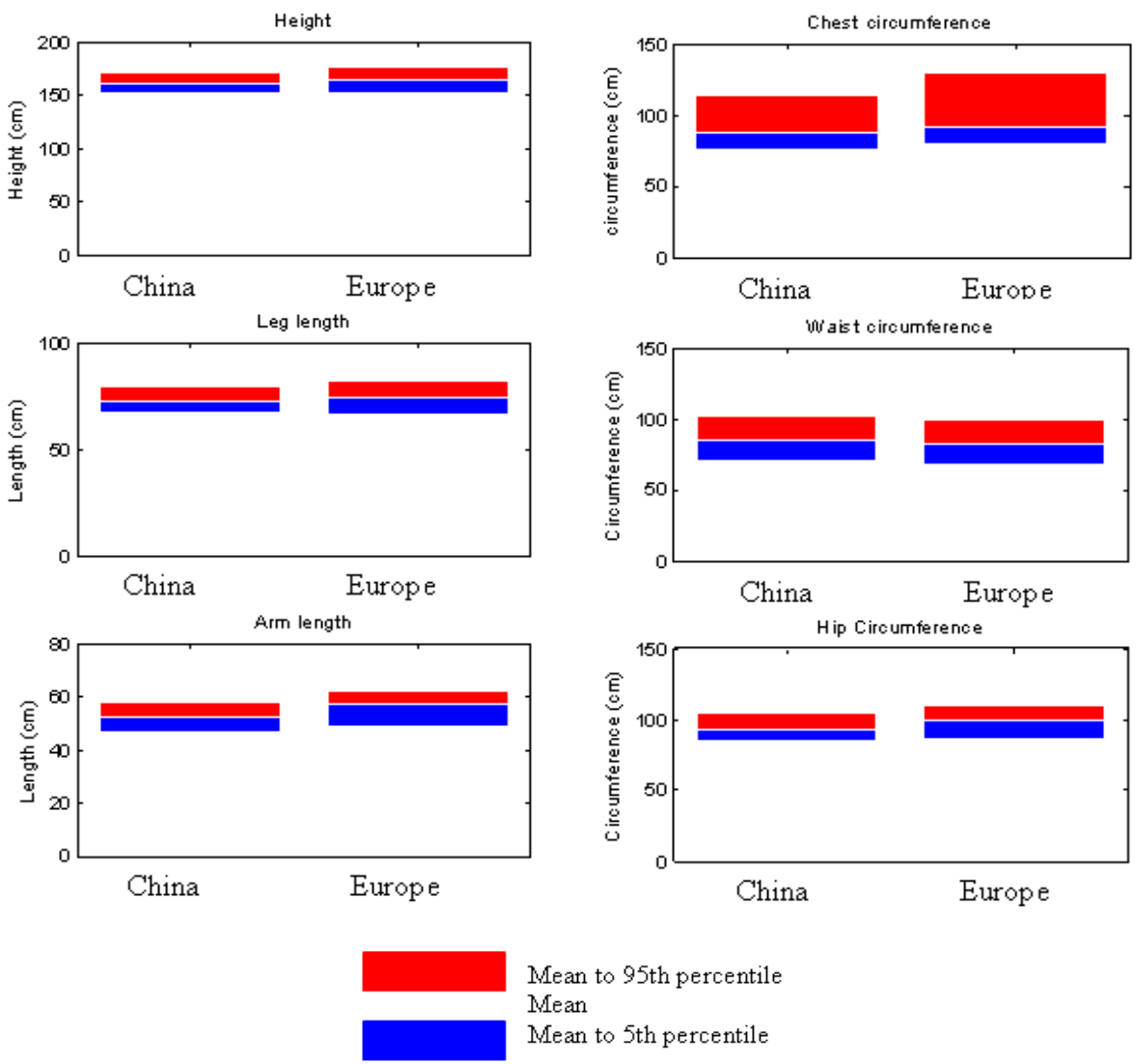

Figure 1: Comparison of female Chinese and European morphology with regards to 8 key measurements 


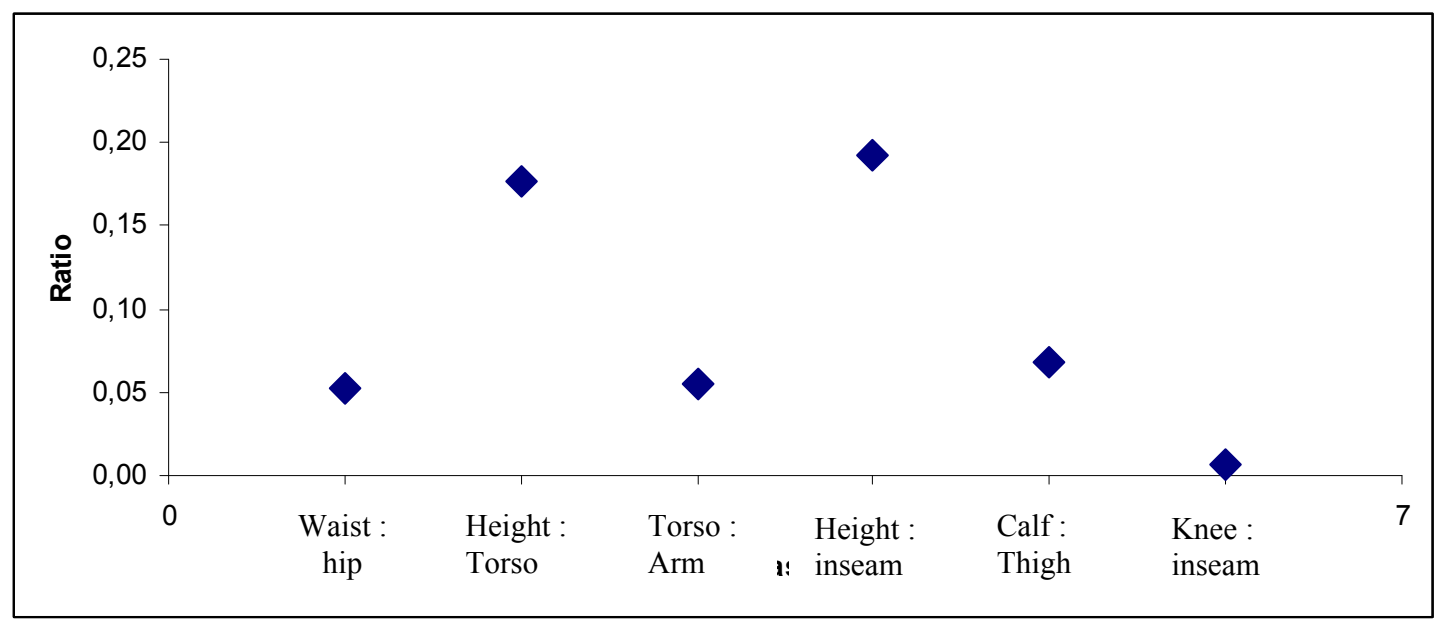

Figure 2: Comparison of absolute difference in ratio between key measurements for European and Chinese women

\section{Discussion and conclusions}

The aim of this paper was to further understand the differences between European and Chinese morphology to determine if separate sizing systems are required to allow for greater population coverage and fit. The results have identified significant differences between the two populations for several key body measures, suggesting that in general the Chinese population are of a smaller frame than Europeans and demonstrate significantly less variation within the population. Although, these results are comprehensive, it needs to be noted that the European population tested was primarily French and Spanish and if a larger study including more countries these differences could be either reduced (inclusion of countries known to be smaller eg. Portugal) or emphasized (inclusion of countries known to be larger eg. Germany and Holland).

As well as overall differences in size, a significant difference in body proportions was found, suggesting that just including smaller sizes would not increase the fit of clothing for the Chinese population, rather a change in shape is required. This was highlighted in the difference in torso and leg length ratios, with the Chinese population being found to have longer bodies and shorter legs than the European population of the same height. As a result of the increase torso length the arm length was found to be comparably shorter despite no significant difference in actual length being found between the 2 populations. Finally, it was also apparent that the silhouette shape of the female population was significantly different, with the ratio between waist and hip circumference being smaller for the Chinese population, indicating a straighter body shape (rectangular) compared to that of the Europeans which typically has a more defined waist (hourglass).

In conclusion these results suggest that to maximise fit and in turn coverage of both populations it is necessary to provide 2 different sizing systems. This is as a result of the differences in populations not only being due to size but also shape. However, further study is required through the incorporation of other countries within Europe, to fully determine if the differences between Asia and Europe are greater than those within Europe and therefore, require a separate sizing system as recommended as a result of this study.

\section{References}

Lin, Y., Wang, M. J. and Wang, E. M. (2004) The comparisons of anthropometric characteristics among four peoples in East Asia,. Applied Ergonomics 35 173-178

Pheasant, S., (1996). Bodyspace: Anthropometry, Ergonomics and Design of Work. Taylor \& Francis, New York, pp. 15-45, 174-193. 
Zakaria, N., Salleh, J., Nasir, M., Yap, T. and Wah, B., (2008) Creating new sizing system for children's wear using anthropometric data. Fifth International Cyberspace Conference on Ergonomics

Emanuel, I., Alexander, M., Churchill, E., Truett, B., (1959). A height-weight sizing system for flight clothing. In: Wright Air Development Center. WADC Technical Report 56-365. Wright-Patterson Air Force Base, Ohio.

Chung,M.J, Lin,H.F. and Wang, M.J.J. (2007) The development of sizing systems for Taiwanese elementary- and high-school students. International Journal of Industrial Ergonomics, Volume 37(8). pp. 707-716.

Tryfos, P. (1986), "An integer programming approach to the apparel sizing problem", Journal of the Operational Research Society, 37, 10, pp. 1001-6.

McCulloch, C.E., Paal, B. and Ashdown, S.A. (1998), "An optimization approach to apparel sizing", Journal of the Operational Research Society, 49, pp. 492-9.

Moon, J.Y. and Nam, Y.J. (2003), "A study the elderly women's lower figure type classification and lower garment sizing systems", Proceedings of International Ergonomics Association Conference, Seoul, Korea.

She, F.H., Kong, L.X., Nahavandi, S., Kouzani, A.Z., 2002. Intelligent animal fiber classification with artificial neural networks. Textile Research Journal 72 (7), 594-600.

Hsu, C.-H. and Wang, M.-J.J. (2005), "Using decision tree-based data mining to establish a sizing system for the manufacture of garments", International Journal of Advanced Manufacturing Technology, 26, 669-74. 